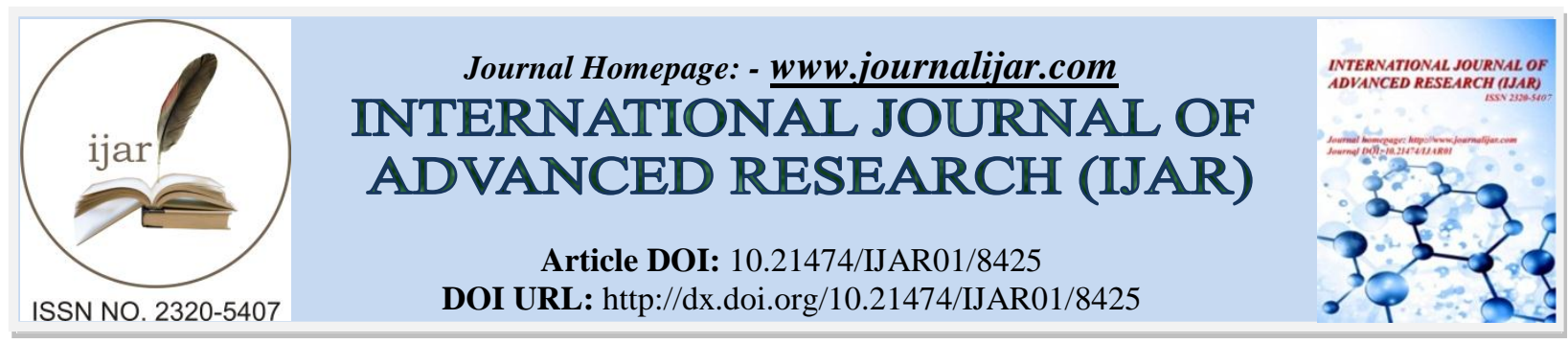

RESEARCH ARTICLE

\title{
MYOCARDITE AIGUE RÉVÉLANT UNE MALADIE DE STILL.
}

A.Benelmekki, M.Malki, I.Asfalou and A.Chaib.

Centre de cardiologie ; Hôpital Militaire d'Instruction Mohammed V - Rabat - Maroc.

\section{Manuscript Info}

Manuscript History

Received: 16 November 2018

Final Accepted: 18 December 2018

Published: January 2019

Key words:-

Still disease, myocarditis, pericarditis, ferritin..

\begin{abstract}
Adult Still disease is an uncommon disorder usually associating high spiking fever, evanescent skin rash constituted of small salmon pink macules, and arthritis. It is characterized by numerous systemic manifestations; a sore throat is common, mobile and indolent lymph nodes, usually in the cervical area. Liver involvement is common and usually limited to a mild or moderate cytolysis. Amongst the other numerous systemic manifestations that have been reported, pericarditis is common, rarely a myocarditis, the pulmonary involvement may lead to an acute respiratory distress, and the rare neurological manifestations include aseptic meningitis or cranial nerve palsy. From a biological point of view the sedimentation rate is consistently elevated and there is usually a marked elevation in the polymorphonuclears. The bacteriological survey is negative as are the immunological tests. It is the increase of the serum level of ferritin and the marked decrease in its glycosylated fraction below $20 \%$ that seem to be of more potent diagnostic value. We report an uncommon case of a 28 -year-old patient admitted for myocarditis whose etiological investigations revealed an adult Still disease.
\end{abstract}

\section{Introduction:}

La maladie de Still de l'adulte est une maladie systémique rare, de cause inconnue, caractérisée par des manifestations cliniques variées pouvant engager le pronostic fonctionnel et/ou vital. Le diagnostic repose sur un ensemble de critères et la prise en charge est non encore codifiée. Elle se manifeste typiquement par une fièvre élevée hectique, des arthralgies ou des arthrites, et une éruption cutanée fugace. Les nombreuses manifestations systémiques qui peuvent diversement s'associer à cette triade rendent compte du polymorphisme de cette affection. Parmi les manifestations cardiaques rapportées, la péricardite est la plus fréquente. De façon exceptionnelle, il s'agit d'une myopéricardite dont le diagnostic est souvent difficile.

\section{Observation:}

Il s'agit d'un patient de 28 ans, qui a été hospitalisé pour dyspnée classe III de la NYHA dont le début de sa symptomatologie remonte à un mois avant son admission par un syndrome algique diffus, une douleur thoracique au repos et une dyspnée classe II de la NYHA, évoluant dans un contexte fébrile. L'examen clinique avait objectivé une tension artérielle à 115/85 mmHg, une fréquence cardiaque à $92 \mathrm{bpm}$, les bruits du cœur étaient assourdies sans souffle ni bruits surajoutés, l'auscultation pulmonaire avait objectivée des râles crépitants aux deux bases pulmonaires, et l'examen abdominal avait trouvé une hépatosplénomégalie, les articulations étaient libres. 
L'électrocardiogramme avait objectivé des troubles de la repolarisation à type d'onde $\mathrm{T}$ négatives en antéroséptal (figure 1). La radiographie pulmonaire de face avait trouvé une légère cardiomégalie avec un index cardiothoracique à 0,6 avec un syndrome interstitiel bilatéral. Le bilan biologique initial avait objectivé une troponine ultrasensible élevée, un syndrome inflammatoire sans point d'appel infectieux (ECBU et hémocultures restées stériles). L'échocardiographie transthoracique (figure $2-3$ ) avait trouvé un ventricule gauche dilaté à $71 \mathrm{~mm}$ de diamètre télédiastolique, une hypokinésie globale avec une fraction d'éjection altérée à $40 \%$, et un strain longitudinal global à $-11,4 \%$, les pressions de remplissage étaient élevées et la pression pulmonaire était à 50 mmHg, l'ETT a objectivé également un épanchement péricardique de faible abondance sans retentissement hémodynamique. L'IRM cardiaque a confirmé le diagnostic de myopéricardite.

Le diagnostic de la maladie de still de l'adulte avec myopéricardite a été retenu devant la symptomatologie clinique, associée à une hyperferritinémie et un effondrement de la fraction glycosylée de la ferritine avec une enquête bactériologique et immunologique négative. Le patient a été mis sous bolus de corticoïdes ( $240 \mathrm{mg}$ de solumedrol) 3 jours de suite avec une nette amélioration clinique et biologique.

\section{Discussion:}

La maladie de Still de l'adulte (MSA) est une affection rare associant de manière variable des manifestations systémiques et articulaires[1-2]. Peu de progrès réels ont été effectués depuis sa description initiale en 1971 par Bywaters[1] et, malgré une meilleure connaissance de la symptomatologie clinique, cette affection, qui survient le plus souvent chez l'adulte jeune, continue à poser des difficultés d'identification, en l'absence d'outil diagnostique de certitude. Seule l'hyperferritinémie et l'effondrement de sa fraction glycosylée semblent avoir un réel intérêt pour le diagnostic [3-4-14].

Au point de vue clinique La MSA se caractérise par une triade associant une fièvre élevée et hectique, une éruption cutanée qui, lorsqu'elle est typique, est évanescente, et une atteinte articulaire à type d'arthralgies ou d'arthrites. À ces manifestations principales peuvent s'ajouter de très nombreuses atteintes systémiques qui peuvent compliquer et parfois obscurcir la présentation clinique, rendant le diagnostic parfois difficile [14]. Sur le plan cardio-vasculaire, la survenue d'une péricardite est fréquente et parfois révélatrice de la maladie [5-6-8-9]. Elle se traduit le plus souvent par une simple douleur thoracique et un frottement péricardique, ou seulement par des anomalies électrocardiographiques, dans certaines observations, l'épanchement péricardique n'est mis en évidence que par une échographie cardiaque effectuée systématiquement. La péricardite est souvent récidivante mais l'évolution vers la constriction n'a été qu'exceptionnellement rapportée [8]. L'atteinte myocardique est plus rare et ne se traduit habituellement que par des troubles de la repolarisation diffuses, des troubles du rythme ou de la conduction, sans grande conséquence clinique [7-8-10-11]. Quelques observations d'atteinte myocardique grave avec insuffisance cardiaque congestive ont été rapportées. Les rares biopsies endomyocardiques pratiquées n'ont alors montré qu'un œdème modéré, une infiltration diffuse par des cellules mononuclées associée par endroit à une prolifération fibroblastique et une hypertrophie modérée de certaines fibres myocardiques [10-11-14]. Une atteinte valvulaire sous la forme d'une insuffisance aortique ou mitrale est exceptionnelle et n'a été rapportée que chez quelques patients seulement, elle a parfois justifié un remplacement valvulaire.

Sur le plan biologique, le syndrome inflammatoire est constant et habituellement très marqué, avec une élévation de la vitesse de sédimentation, de la protéine réactive $\mathrm{C}$ et des autres protéines de l'inflammation [6-7-2]. Il s'accompagne d'une anémie inflammatoire et d'une hypoalbuminémie souvent profondes. De rares observations d'anémie hémolytique parfois à test de Coombs positif ont été publiées [9-12]. Les examens immunologiques sont intéressants par leur négativité, c'est le cas de la sérologie rhumatoïde, des anticorps antinucléaires et anti-DNA natifs, des anticorps anticytoplasme des polynucléaires neutrophiles et de tous les anticorps anti-tissus [7-2-14].

Plusieurs auteurs ont souligné l'intérêt diagnostique de l'hyperferritinémie [3-4-13]. Cette hyperferritinémie pourrait être la résultante d'une activation de cytokines pro-inflammatoires comme l'interleukine 1 (IL-1) et l'IL-6, qui stimulent la transcription de la ferritine. L'élévation de la ferritinémie étant habituelle au cours de tout syndrome inflammatoire, ce dosage n'est cependant réellement utile au diagnostic que lorsque le taux est très élevé. Plus récemment, on a pu montrer que non seulement il existait une élévation de la ferritinémie, mais que celle ci s'associait à un effondrement de la fraction glycosylée en deçà de $20 \%$, le taux normal étant de 60 à $80 \%$ [3-14]. Cette anomalie pourrait avoir une valeur diagnostique plus intéressante, ce d'autant que l'effondrement de la fraction glycosylée semble persister au décours même de la poussée de la MSA malgré une normalisation de la ferritinémie [12]. 
Le traitement reste empirique, reposant sur la corticothérapie ou le methotrexate, et dans les cas réfractaires sur les agents biologiques.

\section{Conclusion:}

Une myopéricardite résistante aux traitements usuels chez un sujet jeune doit faire évoquer le diagnostic de MSA. Cette observation rappelle la gravité potentielle des manifestations cardiaques au cours de la MSA qui peuvent mettre en jeu le pronostic vital.

\section{Conflits d'intérêts :}

Les auteurs ne déclarent aucun conflit d'intérêts

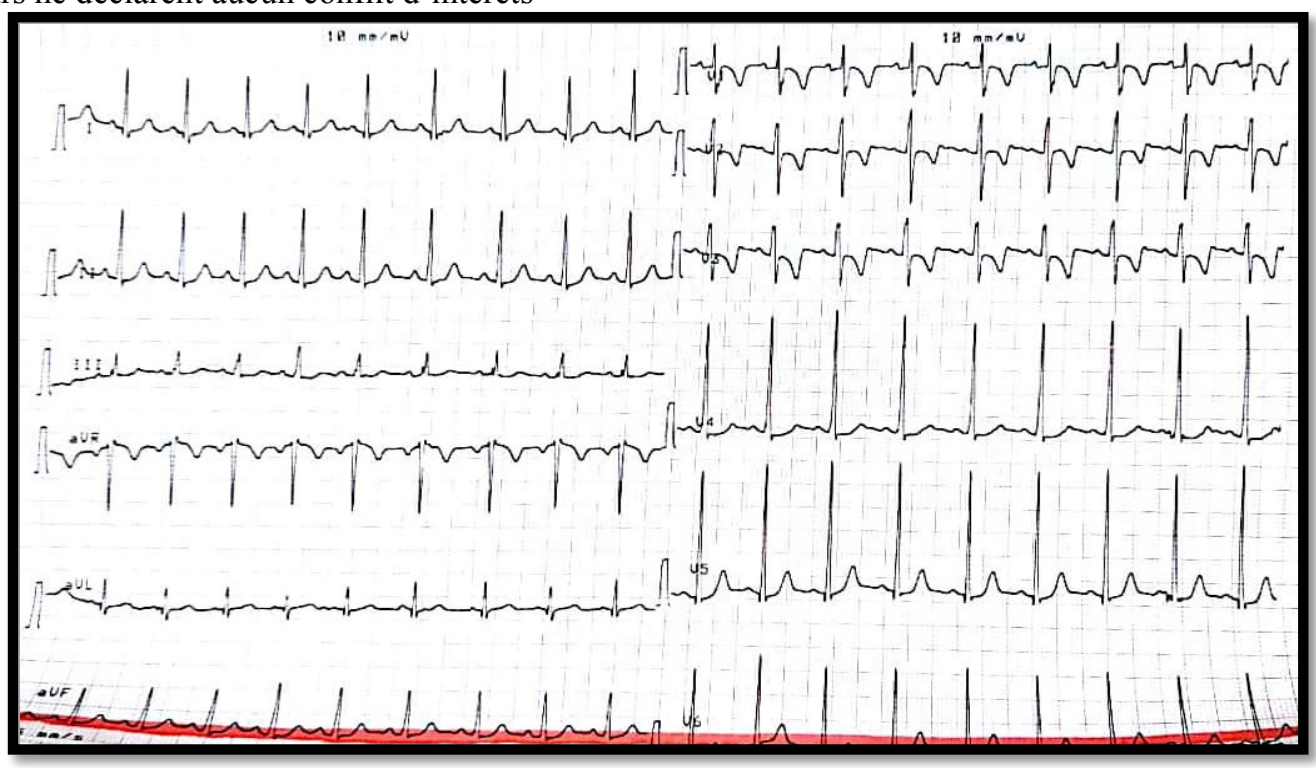

Figure 1: ECG 12 dérivations objectivant les troubles de la repolarisation en antéro-septal

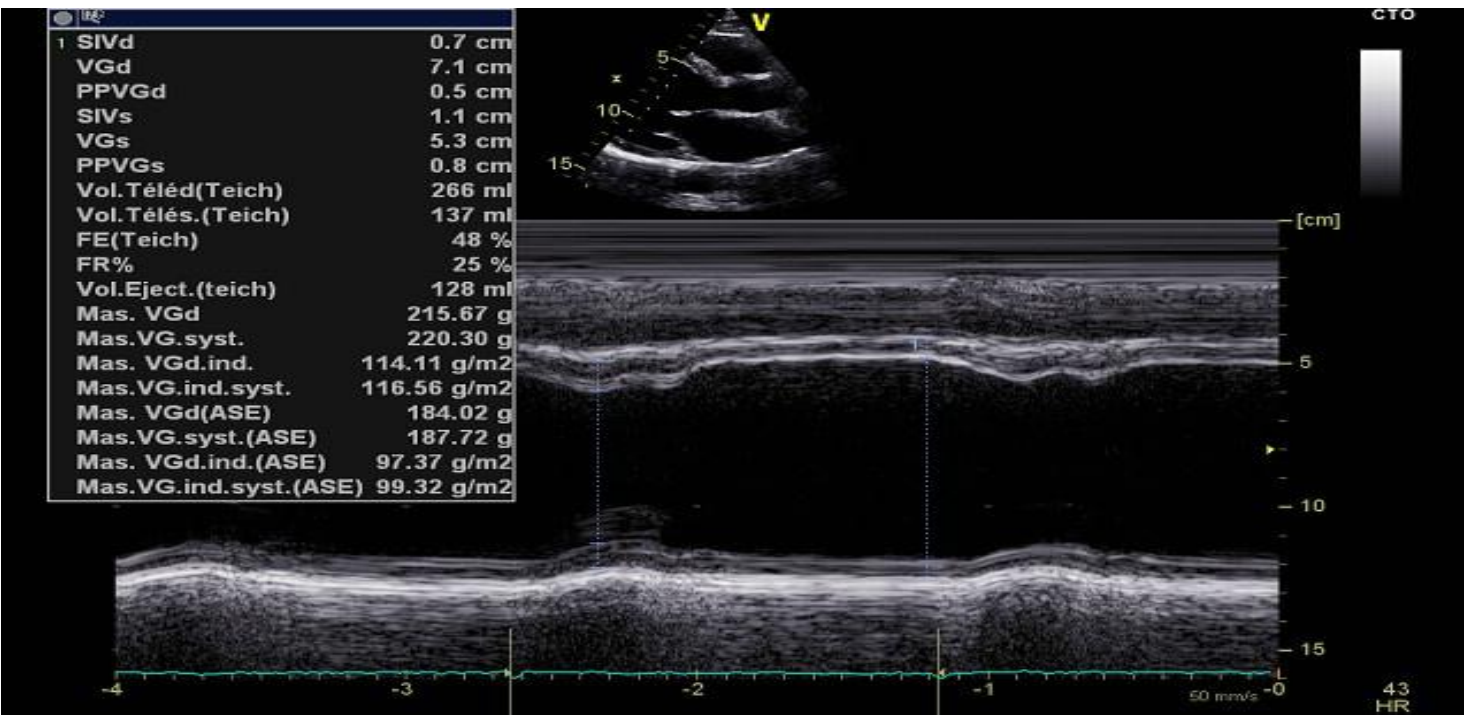

Figure 2: Coupe echocardiographique Temps-mouvement du ventricule gauche 


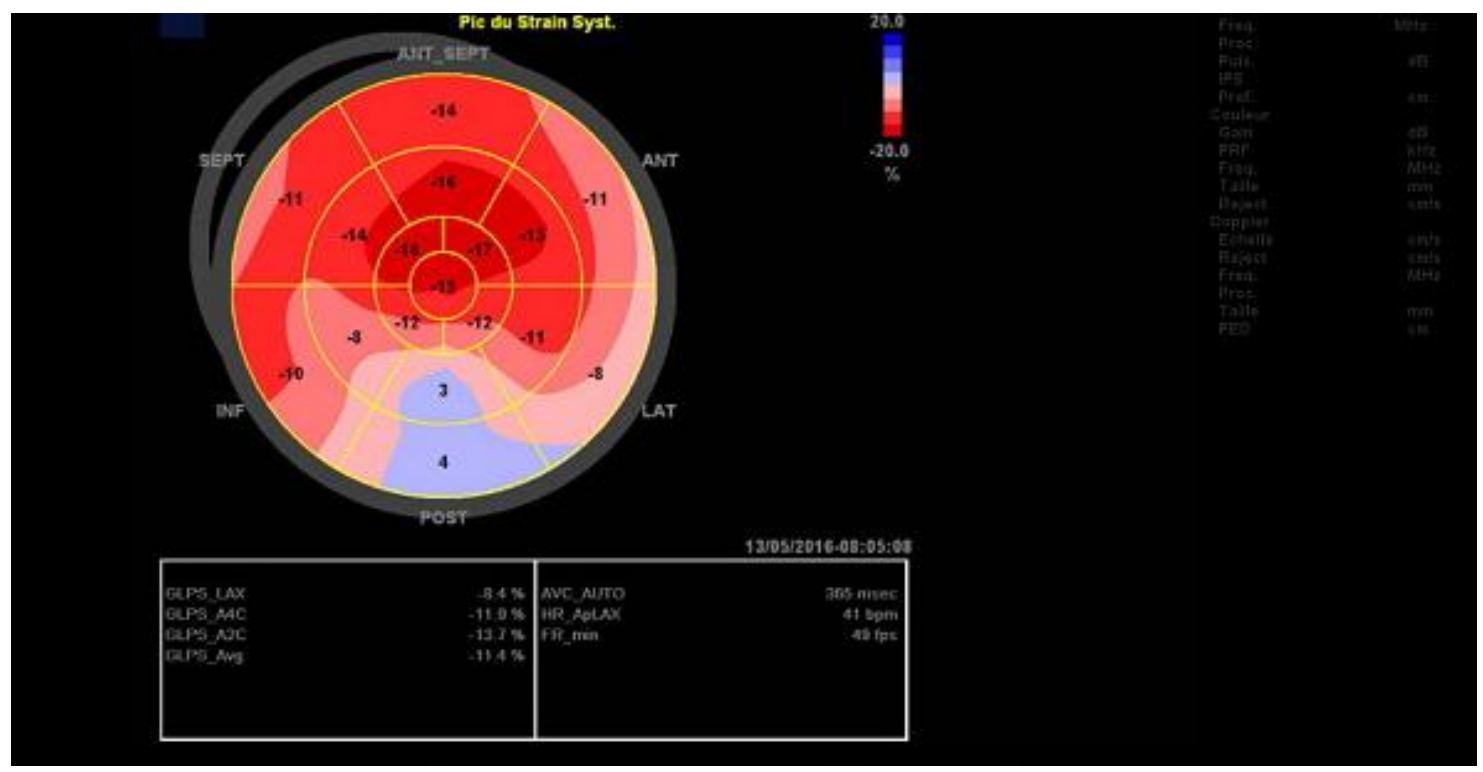

Figure 3: Aperçu œil de bœuf du strain longitudinal global

\section{References:}

1. Bywaters EGL. Still's disease in the adult. Ann Rheum Dis 1971; 30: 121-33.

2. Pouchot J, Kahn MF, Vinceneux Ph. Maladie de Still de l'adulte. In: MF Kahn, AP Peltier, O Meyer, JC Piette. Maladies et syndromes systémiques, 1 vol., 4ème édition 2000, Edition Flammarion Médecine/Sciences, 44968.

3. Van Reeth C, Le Moël G, Lasne Y et al. Serum ferritin and isoferritins are tools for diagnosis of active adult Still's disease. J Rheumatol 1994; 21: 890-5.

4. Fautrel B, Zing E, Golmard JL et al. Proposal for a new set of classification criteria for adultonset Still disease. Medicine 2002; 81: 194-200.

5. Wouters JMGW, van de Putte LBA. Adult-onset Still's disease; clinical and laboratory features, treatment and progress of 45 cases. Q J Med 1986; 235: 1055-65.

6. Reginato AJ, Schumacher HR, Baker DG, O'Connor CR, Ferreiros J. Adult onset Still's disease: experience in 23 patients and literature review with emphasis on organ failure. Semin Arthritis Rheum 1987; 17: 39-57.

7. Ohta A, Yamaguchi M, Kaneoka H, Nagyoshi T, Hiida M. Adult Still's disease: review of 228 cases from the literature. J Rheumatol 1987; 14: 1139-46.

8. Masson C, Le Loët X, Lioté F et al. La maladie de Still de l'adulte. I. Manifestations et complications dans soixante cinq cas en France. Rev Rhum Mal Osteoartic 1995; 62: 805-14.

9. Cush JJ, Medsger JTA, Christy WC, Herbert DC, Cooperstein LA. Adult-onset Still's disease. Clinical course and outcome. Arthritis Rheum 1987; 30: 186-94.

10. Bank I, Marboe CC, Redberg RF, Jacobs J. Myocarditis in adult Still’s disease. Arthritis Rheum 1985; 28 : 452 4.

11. Sachs RN, Talvard O, Lanfranchi J. Myocarditis in adult Still's disease. Int J Cardiol 1990; 27: 377-80.

12. Vignes S, Le Moel G, Fautrel B, Wechsler B, Godeau P, Piette JC. Percentage of glycosylated serum ferritin remains low throughout the course of adult onset Still's disease. Ann RheumDis 2000; 59: 347-50.

13. Coffernils M, Soupart A, Pradier O, Feremans W, Nève P, Decaux G. Hyperferritinemia in adult onset Still's disease and the hemophagocytic syndrome. J Rheumatol 1992; 19: 1425-7.

14. J. Pouchot, P. Vinceneux Manifestations cliniques et biologiques de la maladie de Still de l'adulte Presse Med 2004;33:1012-8. 